\title{
Design of a Smart Model For Geolocalisation and E-commerce in the Semantic Web
}

\author{
Anas Bennani, Samira Khoulji, Kerkeb Mohamed Larbi, \\ Information Systems Engineering Research Group, Dept. Telecommunications. \\ National School of Applied Science of Tetouan, \\ Abdelmalek Essaadi University, Morocco; \\ anasbenn@gmail.com; khouljisamira@gmail.com; kerkebml@gmail.com
}

\begin{abstract}
Currently, e-commerce has become a pillar of the economy; there are huge growths of websites that offer different products to sell.This variety of web portals and products requires intelligent and autonomous operation to successfully the buying / selling process. The semantic web which is an initiation of the inventor of the web Tim Berners-lee proposes advanced technical solutions that act on the organization and the structuration of the data through ontologies.

In this article we will discuss the subject of e-commerce in company with the area of geolocation. This document proposes a new solution allowing taking advantage of the geographical location of a customer to be located in relation to the stores that surround it.
\end{abstract}

Keywords: e-commerce, Semantic Web, GEORSS Model, GEO OWL,OGC GML,Ontlogie FOAF, GoodRelations. Geographic information.

\section{Introduction}

In a world where consumption has become a way of life, the variety of products exposed to sales, the number of shops and supermarkets is increasing, implies the need to put in place computer tools allowing the establishment of a " An optimized policy that completes the whole process: from the presentation of the products to the payment. Actors responding in the field of e-commerce encounter difficulties. On the one hand, the seller must clearly identify his products to customers by specifying all useful information such as price, product description, image, product reviews by other customers who have purchased it.On the other hand, the customer would like to find the product wishing to buy quickly by offering him the necessary information to complete its purchasing operation. It should be noted that these products sold presented on websites, and in the majority of cases, sellers have a real place with an addressin a geographical area, they can sell either online or in their local.

The idea of this research component is to add to the e-commerce process the notion of geographical location. For example, a tourist who finds himself alone in a city that has never visited before, he would like to locate himself in his entourage and find a shop if he exists that sells a particular product, then we are in front of a repetitive scenario for each new visitor, The thing that the ambitious has given us to 
Anas Bennani, Samira Khoulji, Kerkeb Moamed Larbi,. Design of a Smart Model For Geolocalisation and E-commerce in the Semantic Web. Transactions on Machine Learning and Artificial Intelligence, Vol 5 No 4 August (2017); p: 183-189

contribute to improve this problem by proposing a new model allowing the tourism sector or others to benefit.

The word " semantic " in the semantic web domain's specifies the meaning of data on the Web. It is can be discovered by people and also by software. On the other hand, before the appearance of the semantic notion's, a person that read web pages and who create special software those just can infere the senses of the data. The " semantic web " significates an idea witch the software, machines and the person can find, read, understand and use the data on the World Wide Web to achieve useful goals for users. In short, the Semantic Web is supposed to make data situated anywhere on the web accessible and comprehensible by people and by the machine. [1]

It is in this perspective that we are interested in the service of the internet which is the web and more precisely the semantic web which is one of the main emerging actors in this field. Its role is to structure the data via logical links between them.

In parallel we are interested in geographical information (GI) which has become a part of our daily life, especially as we will use it in the GoodRelations ontology which is the vocabulary of E-commerce. The Gl is used as an example to decide the location of a new store or store for the benefit of a company or a trader. More recently, the growth and use of (GI) was exposed by The implementation of Web-based tools such as Google Earth and the creation of GI resources by volunteers, an example being OpenStreetMap, a web-based cartographic resource. The use of geographic information has increased enormously, but many users will be largely unaware of the role it plays in their activities. This is because the $\mathrm{Gl}$ is very rarely an objective in itself;Rather, the scenario on which the topic of interest is remarkable is normally formed.[2]

To create data through the semantic web, we mainly use an ontology[3]. It is a formalization of a conceptualization; it is definition most popular for an ontology. In terms of our semantic web domain, W3C defined an ontology as follows: « An ontology formally defines a common set of terms that are used to describe and represent a domain ... An ontology defines the terms used to describe and represent an area of knowledge. [4]

GeoRSS is a variety of RSS that encodes the geographic location. RSS (Syndication Really Simple or RDF Site Summary) is a web data feed that can be in the form of news reports, quotes or blog topics[5]. Geo OWL presents an ontology that suits the GeoRSS function model. This model will be detailed in a chapter of this document[6].

\section{Existing Ranking Methods}

\subsection{Ontology for E-commerce}

GoodRelations is an ontology dedicated to electronic commerce. It is the strongest vocabulary used to describe products and services. The internet's user can benefit from all the advantages of GoodRelations via the use of his computer, it's able to extract and present the desired product information easily. This ecommerce vocabulary is written by adding codes on web content.[7]

To represent the e-commerce scenario, the Agent-Promise-Object-Compensation model is used. This one is done by 5 entities: An agent is a person or an organization, a promise or offer is a procedure to transfer 
some rights on some object or to provide some service, an object represents product or service, a certain compensation or an amount of money, It is made by the agent and related to the object or service. A location is considered a fifth entity, it determines the place of avaibility of an offer.[7]

The five classes of GoodRelationsare: Gr: BusinessEntity coincides with the agent. Gr: Offering blends with an offer.Gr:PriceSpecification represents the compensation, Gr:ProductOrService for the object or service, Gr: Location represents the five entity. [7]

We are interested in the part of Location whose object of our research. The following image shows the class gr: location and its parameters. [7]

\begin{tabular}{|l|}
\hline \multicolumn{1}{|c|}{ gr:Location } \\
\hline gr:name rdfs:Literal \\
gr:description rdfs:Literal \\
gr:hasGlobalLocationNumber xsd:string \\
gr:hasISICv4 xsd:int \\
gr:category rdfs:Literal \\
\hline
\end{tabular}

Figure 1. Location Class of GoodRelations

\subsection{Ontology for Person}

FOAF ontology "Friend of a Friend" describes people and the relationships they have with each other. It is one of the most popular ontologies in the semantic web. It represents personal information including name, mailbox, home page URL, friends, etc.[8]

FOAF is a dictionary of words that makes a link between people that is used in structured data [8]. It serves to publish web pages for people, groups, companies, etc. This project was founded by Dan Brichleyand Libby Miller. It enriches information about people and their relationships. In this sense, FoaF plays an important role in the creation of information systems that support online communities [9]. Among the [8]personal information that FOAF supports, we can say: FirstName, LastName, SurName, workInfoHomepage,..etc.

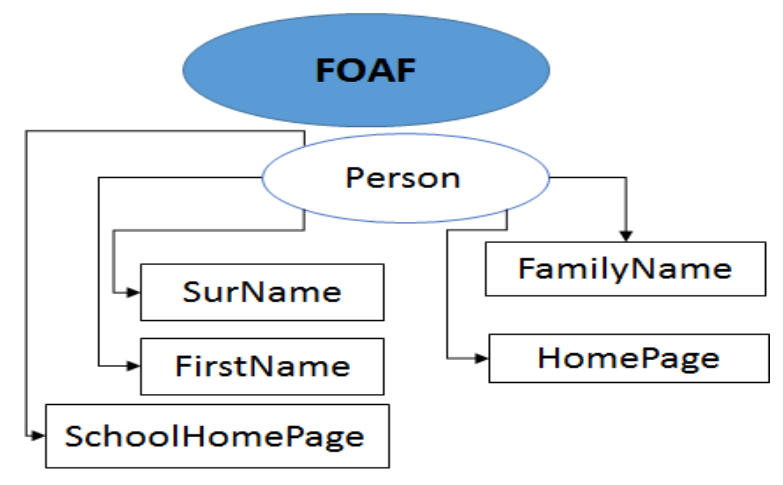

\subsection{GeoRSS Model}

The figure 3 shows the UML design of the GeoRSS model. The left part of the figure shows GeoRSS, the one on the right represents the "external" content that this model uses for its description. The association "Where" links geometry and certain external content. To be able to use this model which is an abstract concept, it must be expressed in XML, RDF, etc. This is called serialization [10].The GeoRSS schema 
Anas Bennani, Samira Khoulji, Kerkeb Moamed Larbi,. Design of a Smart Model For Geolocalisation and

E-commerce in the Semantic Web. Transactions on Machine Learning and Artificial Intelligence, Vol 5 No 4 August (2017); p: 183-189

supports four types of geographic locations: point, line, polygon and Box, they are encoded through a Latitude and Longitude literal string called GeoRSS simple, or through a more robust way using GML called GeoRSSGML[5].

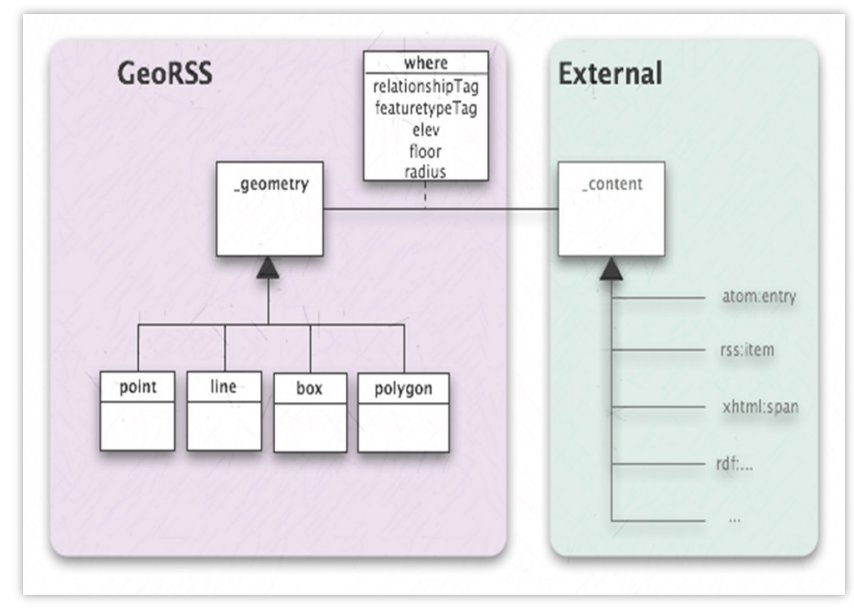

Figure 3. GeoRSS Model

In this document, we will focus on two types: Point and Box that we will define. We will use them in the next chapter.

A point is defined by a pair of coordinates, it contains two values separated by a space:Latitude and after longitude.

A box is defined in two points separated by a space, the first point is the lower corner, which must be far from the second which represents the upper corner, by definition the frame does not contain the North and South poles in its boundary. A box is used to delimit in a rounded way a zone inside.

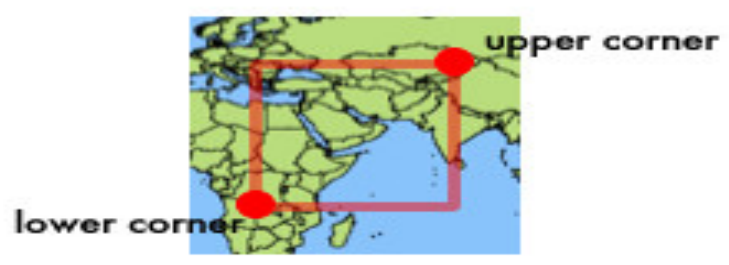

Figure 4. Delimitation of the box type zone

\subsection{GeoRSS and FOAF}

This part of this document shows a basic example that combine the setting of the location of a person through the Geo and FOAF vocabularies. [11] 


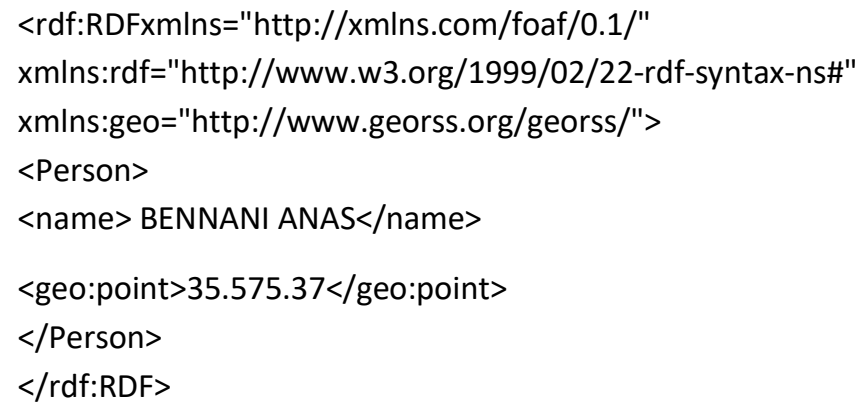

\section{Georss And Goodrelations}

The vocabulary Schema.org is recommended for encoding the geo position, this time the coding below shows how to combine geo with GoodRelations ontology (GR) while using shema.org [12].

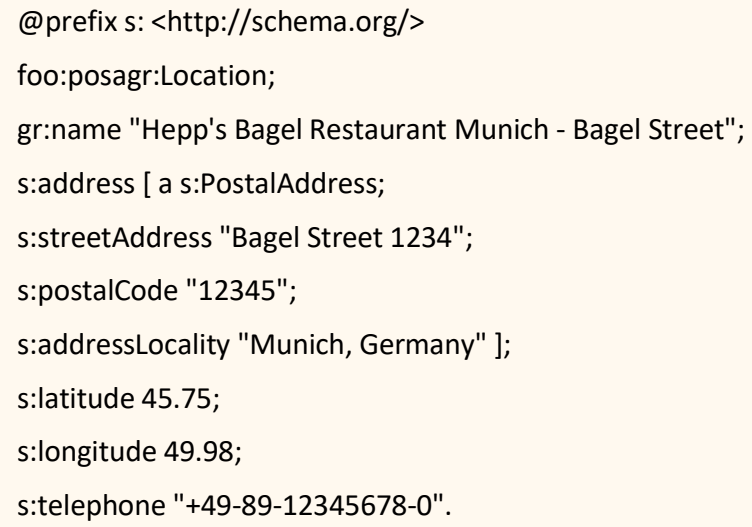

\subsection{A New GeoRSS model approach}

The objective of the model that we implement in this paragraph is to give a tourist or other person connected through a geographic information system the possibility of positioning in a region. And this is affordable by adding a parameter that indicates the desired margin. This parameter is represented in decimal degrees. And therefore all GPS coordinates are expressed by the same unit.

As a legitimate scenario for this approach, a tourist doesn't know enough about the interesting areas of the city she visits, he would like to know if a perfume store or clothing is in his entourage at a precise distance. So this model will answer this problem by representing the differents steps From conception to implementation.

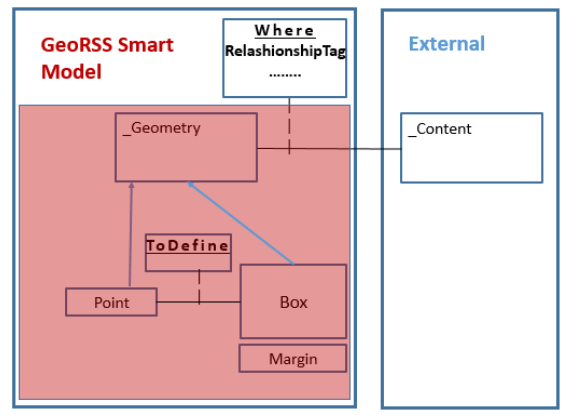

Figure 5. GeoRSSSmart Model 
Anas Bennani, Samira Khoulji, Kerkeb Moamed Larbi,. Design of a Smart Model For Geolocalisation and E-commerce in the Semantic Web. Transactions on Machine Learning and Artificial Intelligence, Vol 5 No 4 August (2017); p: 183-189

Let us say that the latitude position of the person is expressed by PLat and its positition longitude is PLong. The distance will be expressed by the parameter Margin, the Box propertyas shown in the figure below will have the following values: PLat-Margin PLong-Margin representing lower corner, and PLat + Margin PLong + Margin representing upper corner. As you notice the Box property will be implemented in an automatic way, this algorithm makes it easier for the person to know the main places that surround them and he can visit them and take advantage of their opportunities.

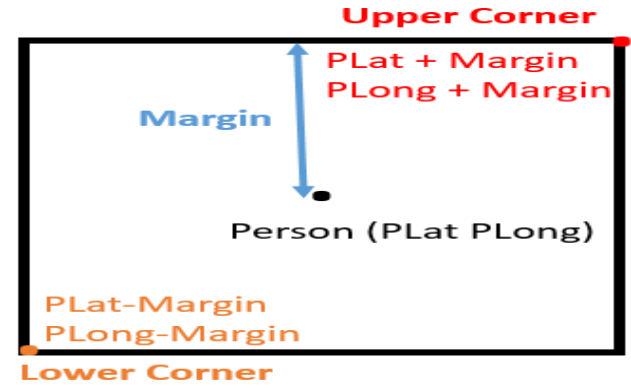

Figure 6.Definition of the Box property area

\subsection{RDF Graph For Model}

In this section, we will present the RDF graph of the new GEORSS model.

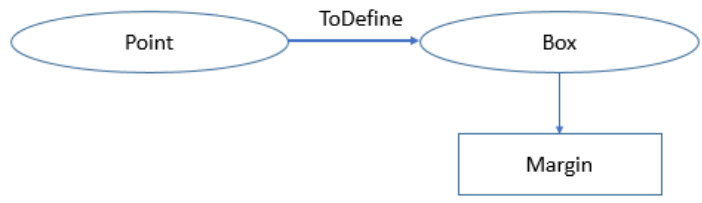

Figure 7. RDF Graph ForGeoRSS Smart Model

\subsection{Implementation of a new model in a semantical way}

To represent the knowledge built on the basis of the RDF model above, we opt for the OWL language: Web Ontology Language.[13]

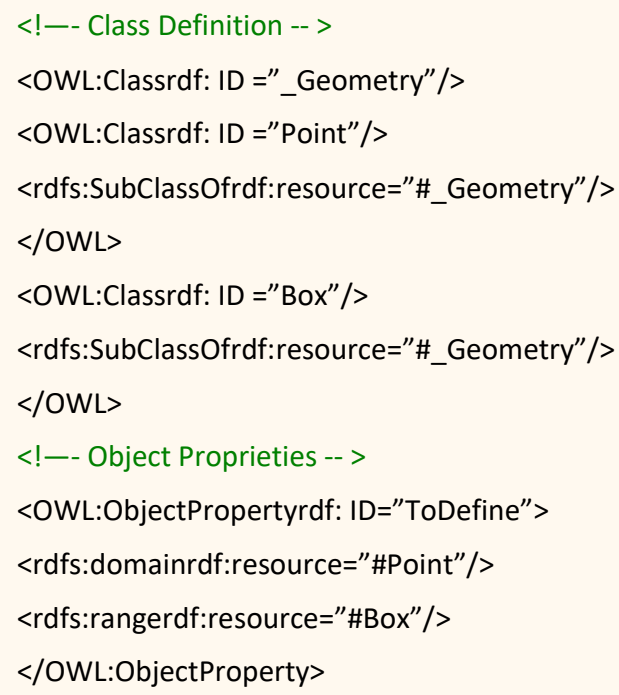


<!-- Data Type Proprieties -- >

<OWL:DataTypePropertyrdf:ID="Margin">

<rdfs:domainrdf:resource="\#Point" $/>$

<rdfs:rangerdf:resource="\#Box"/>

$<$ rdfs:rangerdf:resource="_\&xsd;float" $/>$

$</ O W L: D a t a T y p e P r o p e r t y>$

\section{Conclusion}

In this research component, we focus on the semantic web applied to e-commercein association with geographic information. The goal is to make the electronic commerce smarter allowing to any Internet user to carry out automatically a complex commercial operation with a minimum of interventions.

The internet users become more attached to technology, which causes their behavior change quickly, and the addition of the geo-location component; which has a direct relationship with Internet users; in the field of e-commerce participates in its progress in an accelerated manner.

\section{REFERENCES}

[1] THOMAS B.PASSIN, “Explorer's Guide of the Semantic Web”, Manning Publication Co, ISBN 1-932394-20-6 p3,2004, pp. 3.

[2] GLEN HART,CATHERINE DOLBEAR, Ebook “Linked Data A Geographic Perspective", ISBN: 13: 978-1-4398-6997-0, 2013.

[3] GRIGORIS ANTONIOU, FRANK VAN HARMELEN, "A semantic web primer", FlteBook,The MIT Press Cambridge Massachusetts, London, England 2004.

[4] LIYANG YU, “A developer Guide to the semantic web”, Springer, ISBN : 9783642159695,pp. 136,137, 2011.

[5] LEO ORBST,TERRY JANSSEN,WERNER CEUSTERS, “Ontologies and Semantic Technologies for Intelligence”, IOS Press, ISBN: 9781607505808, p 196, 2010.

[6] Official web site: https://www.w3.org/2005/Incubator/geo/XGR-geo-20071023/

[7] Official web site Martin Hepp, GoodRelations Official Web Site: http://www.heppnetz.de/projects/goodrelations/

[8] Friend of a Firend Project Official web site,Http://www.foaf-project.org/

[9] LI DING, LINA ZHOU, TIM FININ, ANUPAM JOSHI, "How the Semantic Web is Being Used:An Analysis of FOAF Documents" System Sciences, 2005. HICSS '05. Proceedings of the 38th Annual Hawaii International Conference on (2005), pp. 113c-113c

[10] Official web site http://www.georss.org/model.html

[11] https://www.w3.org/2005/Incubator/geo/XGR-geo-20071023/\#examples

[12] http://wiki.goodrelations-vocabulary.org/Documentation/Geo_data

[13] STEPHEN CRANEFIELD, "UML and the Semantic Web", University of Otago, February 2001 